\title{
Processamento auditivo: marcadores de tempo por habilidade auditiva
}

\section{Auditory processing: time markers for hearing ability}

\author{
Marcela B. Deveikis'; Erika B. Mantello²; Patricia P. Mandrá ${ }^{3}$; Myriam L. Isaac ${ }^{3}$; Monica P. Castro ${ }^{4}$, \\ Ana Cláudia M. B. Reis ${ }^{3}$
}

\begin{abstract}
RESUMO
Modelo de estudo: estudo descritivo e transversal. Objetivo do estudo: caracterizar o tempo de aquisição de cada habilidade auditiva trabalhada em crianças diagnosticadas com Distúrbio do Processamento Auditivo Central, em idade escolar, a partir de um programa de intervenção fonoaudiológica pré-estabelecido, com duração máxima de 20 sessões. Metodologia: foram selecionados, para a intervenção fonoaudiológica, 8 indivíduos na faixa etária de sete a nove anos, em idade escolar, de uma escola pública e que completasse, no mínimo, 16 sessões de terapia, ou, ainda, aquele que atingisse os objetivos propostos (adequação das habilidades) em menor número de sessões. Para cada sessão, que ocorreu semanalmente, com duração de 40 minutos, foi realizado registro cursivo do desempenho da criança, para cada uma das habilidades trabalhadas (localização, discriminação, reconhecimento, figura-fundo/ fechamento, compreensão e memória). Posteriormente, foi realizada análise do conteúdo descritivo por semelhança entre as habilidades. No registro, foi assinalada a sessão considerada como término da atividade, isto é, a aquisição da habilidade. Resultados: o número de sessões realizadas pelos indivíduos do estudo variou de 10 a 20 sessões, sendo que o tempo médio de sessões para a aquisição de cada habilidade auditiva trabalhada oscilou entre três e 16 sessões. Conclusões: foi possível observar adequação das habilidades auditivas em todos os indivíduos do estudo, com variação de tempo para cada habilidade trabalhada. Estudos com maior casuística são fundamentais para que se alcancem marcadores de tempo para aquisição de habilidades auditivas, nesse segmento populacional.
\end{abstract}

Palavras-chave: Percepção Auditiva. Reabilitação. Audição. Transtornos da Percepção Auditiva.

\begin{abstract}
Model of study: descriptive study and cross. Purpose: to characterize the acquisition time of each hearing ability worked with children diagnosed with Disturbance of Central Auditory Processing, school age, from a speech therapy program pre-established, which lasted 20 sessions. Methods: participated in speech therapy 8 patients aged seven to nine years, school age, in a public school. For the analysis of the data set as a criterion for participation, the individual who completed at least 16 sessions of therapy, or one that
\end{abstract}

1. Fonoaudióloga graduada pela Faculdade de Medicina de Ribeirão Preto da Universidade de São Paulo (FMRP-USP), Ribeirão Preto, São Paulo, Brasil.

2. Fonoaudióloga Doutora, assistente do curso de Fonoaudiologia da FMRP-USP.

3. Docentes do Departamento de Oftalmologia, Otorrinolaringologia e Cirurgia de Cabeça e Pescoço da FMRP-USP.

4. Fonoaudióloga Doutora, docente da Universidade de Franca, Franca, São Paulo, Brasil.

Correspondencia Erika Barioni Mantello Curso de Fonoaudiologia da FMRP-USP. Av. Bandeirantes, 3600, Campus Universitário. CEP: 14049-900. / Ribeirão Preto/ SP

Artigo recebido em 11/09/2014 Aprovado para publicação em 20/02/2015 
reached the proposed objectives (adequacy of skills) in fewer sessions. For each session, which occurred weekly, lasting 40 minutes each, was conducted cursive record the child's performance for each of the skills worked (location, discrimination, recognition, figure-ground/closing, comprehension e memory). Subsequently, we performed a descriptive content analysis of similarity between the skills. In the record was marked session considered the end of the activity, that is, the acquisition of skill. Results: the number of sessions held by the study subjects ranged from 10 to 20 sessions and the average length of sessions for each skill acquisition of auditory worked varied from three to 16 sessions. Conclusion: it was possible to observe adequacy in auditory skills in all subjects of the group, with a range of time worked for each skill. Studies with larger samples are essential to achieve time markers for the acquisition of auditory skills in this population segment.

Keywords: Auditory Perception. Rehabilitation. Hearing. Auditory Perceptual Disorders .

\section{Introdução}

O Processamento Auditivo Central (PA) pode ser definido como uma série de operações mentais que o indivíduo realiza ao lidar com as informações sonoras recebidas e que dependem da capacidade biológica inata do organismo e da experienciação no meio acústico. ${ }^{1}$ A manipulação e utilização dos sinais sonoros pelo Sistema Nervoso Auditivo Central (SNAC): "o que fazemos com o que ouvimos" 2 é outra definição do PA.

Pesquisadores afirmaram que o processo de recepção e integração do sinal acústico é possível porque o sistema auditivo desempenha as seguintes habilidades: detecção do som, localização, atenção, figura-fundo, síntese ou integração binaural, separação binaural, fechamento, reconhecimento, discriminação, compreensão ou cognição e memória. ${ }^{3-6}$

Indivíduos cujo histórico sugerir dificuldade de compreensão, em ambientes com barulho, reverberação em situações de grupo, bem como dificuldade de localização sonora, de memória e até mesmo distração e/ou insucesso em terapia fonoaudiológica, sem a presença de perda auditiva importante, podem apresentar Distúrbio do Processamento Auditivo Central (DPA). ${ }^{7}$

Zalcman e Schochat ${ }^{8}$ descreveram o DPA como uma alteração da audição na qual há impedimento da habilidade de analisar e/ou interpretar padrões sonoros, podendo ser resultado de prejuízo biológico inato ou da falta de experiências em um ambiente acústico. Depois de constatado o DPA, alguns procedimentos são propostos na tentativa de minimizar as habilidades auditivas alteradas, entre eles o Treinamento Auditivo (TA), que é considerado um conjunto de estratégias utilizadas para desenvolver ou reabilitar as habilidades auditivas, necessárias para a compreensão da fala, bem como orientação a pais, educadores e a melhora do ambiente acústico.

Segundo Grafman ${ }^{9}$ e Tremblay e Kraus ${ }^{10}$, os programas de TA podem melhorar as funções auditivas em decorrência da plasticidade neural, definida como modificação em células nervosas que ocorrem a partir de influências ambientais, como a prática de uma habilidade, ou exposição frequente a um estímulo, e que causariam uma mudança comportamental.

De acordo com Zalcman e Schochat ${ }^{8}$, as características gerais de um programa de TA eficaz contém, como fatores essenciais, ser intensivo, conter atividades desafiantes ao sistema auditivo e suficientemente interessante, de modo a manter a motivação do paciente, evitando sua frustração. A terapia deve fornecer à criança oportunidade para aprender a escutar com atenção e a processar os estímulos verbais, para que possa compreender e interpretar a conversação em diferentes ambientes e situações. A criança deve aprender a usar sua audição para compreender a fala de outros, monitorar a própria fala e os sons ambientais. ${ }^{11}$

Desse modo, o TA e outras formas de intervenção comportamental que promovam a estimulação auditiva necessitam de tempo para desenvolver ou reabilitar as habilidades auditivas alteradas, necessárias para a compreensão da fala. Balizadores de tempo de tratamento fonoaudiológico constituem parâmetros importantes para o planejamento, acompanhamento e avaliação de serviços de saúde. Na perspectiva de aprimorar o exercício profissional, impõe-se, para toda a classe fonoaudiológica, o desafio de refletir, debater e estabelecer consensos sobre esses parâmetros, sempre tendo como base o coletivo e visando o planejamento, controle e monitoramento dos serviços. 
O estabelecimento dos balizadores, entretanto, não deve modificar ou substituir a conduta profissional, que é norteada pelo conhecimento, experiência e pela situação específica do paciente em atendimento. ${ }^{12}$

Não foram encontrados na literatura estudos específicos sobre o tempo de terapia propostos nos casos de DPA, embora em alguns trabalhos publica$\operatorname{dos}^{13-19}$ o número variasse de oito a 18 sessões de TA.

O objetivo neste estudo foi caracterizar o tempo de adequação de cada habilidade auditiva trabalhada em crianças com diagnóstico de DPA, em idade escolar, a partir de um programa de intervenção fonoaudiológica pré-estabelecido, com duração máxima de 20 sessões.

\section{Material e Métodos}

A presente investigação consistiu em estudo descritivo e transversal, com amostra não probabilística.

Este trabalho foi autorizado pelo Comitê de Ética em Pesquisa, de acordo com o Processo no 2081/ 2008. Os responsáveis pelas crianças autorizaram a participação destas, mediante assinatura do Termo de Consentimento Livre e Esclarecido, após terem recebido orientação sobre a pesquisa.

Os critérios de inclusão para realização do TA foram: que os sujeitos apresentassem avaliação audiológica (audiometria tonal limiar, logoaudiometria, imitanciometria), com resultados dentro dos padrões de normalidade, ausência de queixas atuais ou anteriores de afecções do sistema auditivo e, ainda, apresentassem avaliação do processamento auditivo, identificando alteração de habilidades auditivas. Além disso, a criança deveria completar, no mínimo, 16 sessões de terapia, ou atingir os objetivos propostos (adequação das habilidades) em menor número de sessões.

Foram excluídas crianças com perda auditiva periférica, com alterações psicológicas e neurológicas e que não completassem o mínimo de sessões estipulado. No serviço, onde essas crianças estavam em atendimento para diagnóstico médico previamente ao processo de intervenção fonoaudiológica, realiza-se avaliação pela equipe multidisciplinar (médico, psicólogo, fonoaudiólogo), o que permitiu a seleção da amostra.

Foram selecionadas oito crianças com diagnóstico de DPA, na faixa etária de sete a nove anos, que frequentavam ensino fundamental de uma escola pública do município de Ribeirão Preto, SP. O Treinamento Auditivo foi realizado no ambulatório de Otorrinolaringologia e Fonoaudiologia da instituição de origem, num período de cinco meses, perfazendo o total de 20 sessões, realizadas semanalmente, com duração de 40 minutos cada.

A avaliação do processamento auditivo e o treinamento das habilidades foram realizados por um fonoaudiólogo.

A avaliação comportamental da função auditiva central foi constituída por testes de estímulos verbais e não verbais, realizados em cabina acústica, por meio de audiômetro de dois canais, modelo Midimate 622 da marca Madsen, acoplado a um CD Player da marca Sony com fones TDH 39, que permite a apresentação dos estímulos por meio de Compact Disc. Os Compact Discs utilizados foram os que acompanham o livro Processamento Auditivo Central - Manual de Avaliação, volumes 1 e 2 para os testes dicótico de dígitos, teste de Sentenças Sintéticas com mensagem competitiva (SSI) e teste dicótico consoante-vogal. ${ }^{20}$

O TA baseou-se em apresentar experiências intensivas que pudessem desafiar o sistema nervoso auditivo central a realizar atividades cada vez mais complexas, por vezes associadas a outros sistemas (por exemplo: visual, motor) com o objetivo de provocar modificações no comportamento auditivo de cada criança.

Durante as sessões de TA, os indivíduos receberam estímulos auditivos verbais e não verbais, por meio do fone de ouvido, a fim de estimular as habilidades auditivas de discriminação, figura-fundo, compreensão e memória auditiva (resolução temporal e ordenação temporal), dos programas "Processando Sons" ${ }^{21}$, material em CD de audio, com 29 exercícios de estimulação auditiva, dividido em oito partes, e "Pedro na casa mal-assombrada" 22 , que consiste em um software específico para desenvolver as habilidades de processamento auditivo, composto por oito exercícios, envolvendo diferentes padrões verbais e não verbais.

Os objetivos foram definidos e as atividades variaram em níveis de complexidade progressivos durante o processo de intervenção, iniciou-se com tarefa mais fácil em termos de estímulo auditivo até chegar ao estímulo auditivo mais difícil.

As habilidades auditivas avaliadas e identificadas como alteradas foram trabalhadas formal e in- 
formalmente, seguindo critérios de complexidade do treino, ou seja, de simples para complexo e apresentação do estímulo em conjunto fechado para o aberto, sem e com som competitivo, ordenação (sequência) e percepção de intervalos.

As habilidades auditivas trabalhadas e as atividades propostas no programa de intervenção fonoaudiológica foram:

- localização - variaram-se estímulo e posição da fonte sonora;

- discriminação/reconhecimento/fechamento - variaram-se o estímulo e a intensidade, com e sem estímulos competitivos em diferentes relações de sinal/ruído;

- atenção seletiva/figura-fundo - foi trabalhada com estratégias que viabilizassem a identificação de sons (ambientais e de fala) em situações competitivas (relação sinal/ruído com sons instrumentais, ambientais, musicais, histórias e burburinhos);

- processamento temporal - por meio de atividades lúdicas, com variações de frequência, intensidade e duração (sons breves e sucessivos), ordenação (sequência) e percepção de intervalos.

O tempo determinado, em número de sessões de terapia, para adequação de cada habilidade auditiva, foi baseado em pesquisas publicadas ${ }^{8,13-19}$, e nos registros de prontuários de pacientes com DPA em alta.

O desempenho do indivíduo para cada tarefa foi considerado em porcentagem, por exemplo, em uma atividade que envolvesse uma tarefa com 10 respostas diferentes, cada uma corresponderia a $10 \%$. Para este estudo, considerou-se como desempenho mínimo, para passar ao nível de complexidade seguinte, um resultado maior ou igual a $80 \%$ e para diminuir a complexidade da tarefa, o resultado deveria ser inferior a $30 \%{ }^{23}$ Segundo Musiek e Schochat ${ }^{24}$ as estratégias de estimulação devem desafiar o sistema nervoso para favorecerem a aprendizagem, portanto, não devem ser nem muito fáceis nem muito difíceis.

Para cada sessão foi realizado registro cursivo do desempenho do indivíduo para cada habilidade trabalhada, em planilha previamente elaborada. Considerou-se como critério de adequação da habilidade trabalhada, como descrito anteriormente, quando a criança obtinha percentual $\geq 80 \%$ de acerto. Posteriormente, foi realizada análise do conteúdo por semelhança das habilidades trabalhadas. No registro diá- rio de cada criança, foi assinalada a sessão considerada como término da atividade, isto é, a adequação da habilidade.

O estudo estatístico constituiu-se de análise exploratória dos dados, foi realizada análise descritiva da distribuição de frequência de todas as variáveis categóricas e análise das medidas de tendência central e de dispersão das variáveis contínuas, com o objetivo básico de sintetizar uma série de valores de mesma natureza, permitindo que houvesse visão global da variação desses valores, organizando e descrevendo por meio de tabela e figuras com medidas descritivas. Essa análise foi realizada com o auxílio software SAS ${ }^{\circledR}$ 9.0, utilizando a PROC MEANS.

\section{Resultados}

Foram incluídas para a presente análise oito crianças com idade entre sete e nove anos, com média de oito anos de idade, que tiveram adequação de todas as habilidades trabalhadas durante o programa de TA, com no mínimo de 16 sessões de treinamento auditivo ou em menor número de sessões. Neste estudo apenas o sujeito 6 completou em 10 sessões (Figura 1). Dessas crianças, três eram do sexo feminino e cinco do sexo masculino.

Na Tabela 1, observa-se o tempo médio de sessões para adequação de cada habilidade auditiva trabalhada nos indivíduos no estudo $(\mathrm{N}=8)$.

Na Figura 1, observa-se a distribuição dos indivíduos por número de sessões realizadas $(\mathrm{N}=8)$ e tipo de habilidade treinada.

Na Figura 2, observa-se a distribuição do número de sessões para aquisição das habilidades auditivas, por indivíduo $(\mathrm{N}=8)$.

Todos os indivíduos estudados atingiram a adequação das habilidades auditivas propostas, com variação de tempo para cada habilidade trabalhada.

\section{Discussão}

No programa de TA realizado, considerou-se a importância do registro e sistematização do processo terapêutico para verificar quais variáveis poderiam influenciar a reabilitação e quais seriam as diretrizes para o redirecionamento da intervenção. A caracterização da amostra que participou da intervenção comportamental fonoaudiológica foi composta por oito indivíduos, sendo a maioria $(62,5 \%)$ do sexo masculino. Um estudo consultado refere que o DPA é mais 
Tabela I: Média do número de sessões necessárias para adequação das habilidades auditivas

\begin{tabular}{lcccccc}
\hline Habilidades Auditivas & N & Média & Desvio Padrão & Mínimo & Mediana & Máximo \\
\hline Localização & 8 & 2,87 & 1,64 & 1 & 3,5 & 5 \\
Discriminação - fonêmica & 8 & 6,5 & 2,33 & 4 & 6 & 10 \\
Discriminação - pares mínimos & 8 & 9 & 1,93 & 5 & 10 & 10 \\
Reconhecimento & 8 & 16 & 4,41 & 6 & 17 & 20 \\
Figura-fundo/fechamento & 6 & 4,66 & 0,81 & 3 & 5 & 5 \\
Compreensão & 8 & 12,25 & 1,75 & 10 & 12 & 15 \\
Memória - sem som competitivo & 8 & 5 & 0 & 5 & 5 & 5 \\
Memória - com som competitivo & 8 & 6,5 & 2,67 & 2 & 6,5 & 10 \\
\hline
\end{tabular}

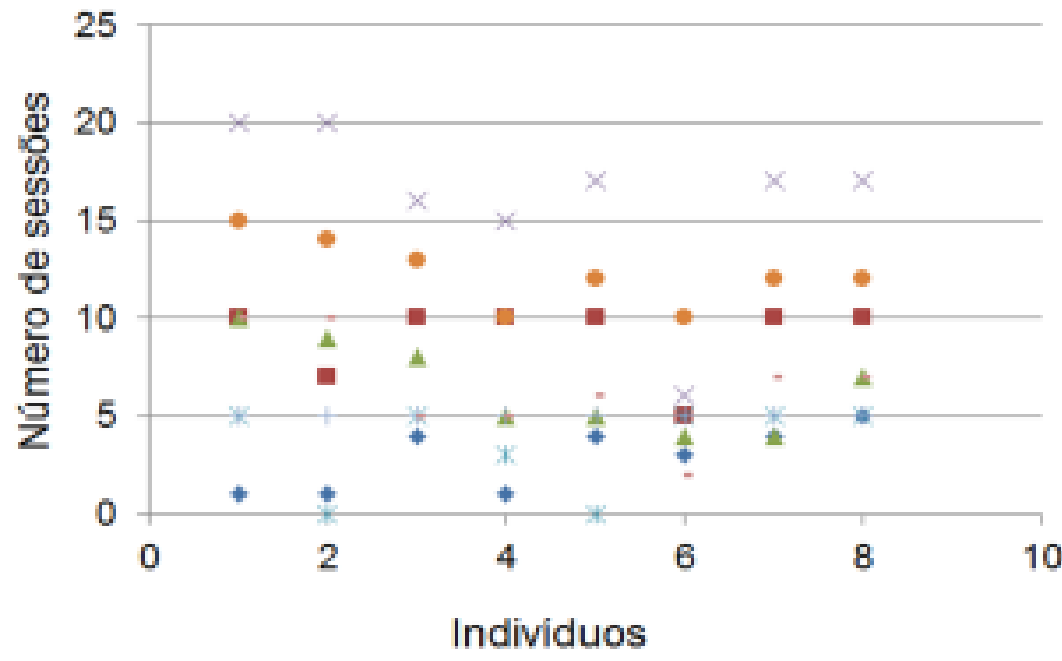

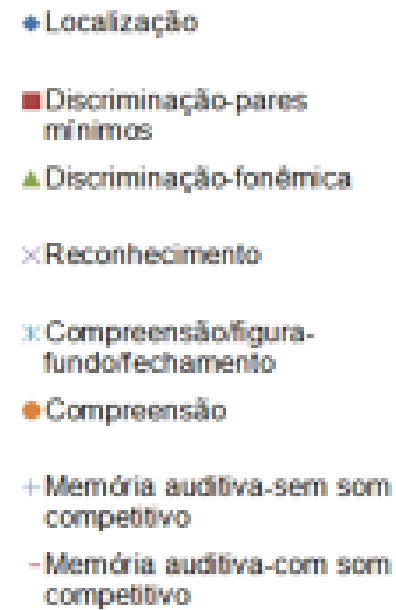

- Localizaçăo

Discriminaçềo-pares minimas

Discriminaçäo-fonêmica

Reconhecimento

x. Compreensàolfigura-

fundoifechamento

- Compreensắ

Memória audiova-sem som competovo

competiovo

Figura 1: Distribuição dos indivíduos por número de sessões realizadas $(\mathrm{N}=8)$ e tipo de habilidade treinada.

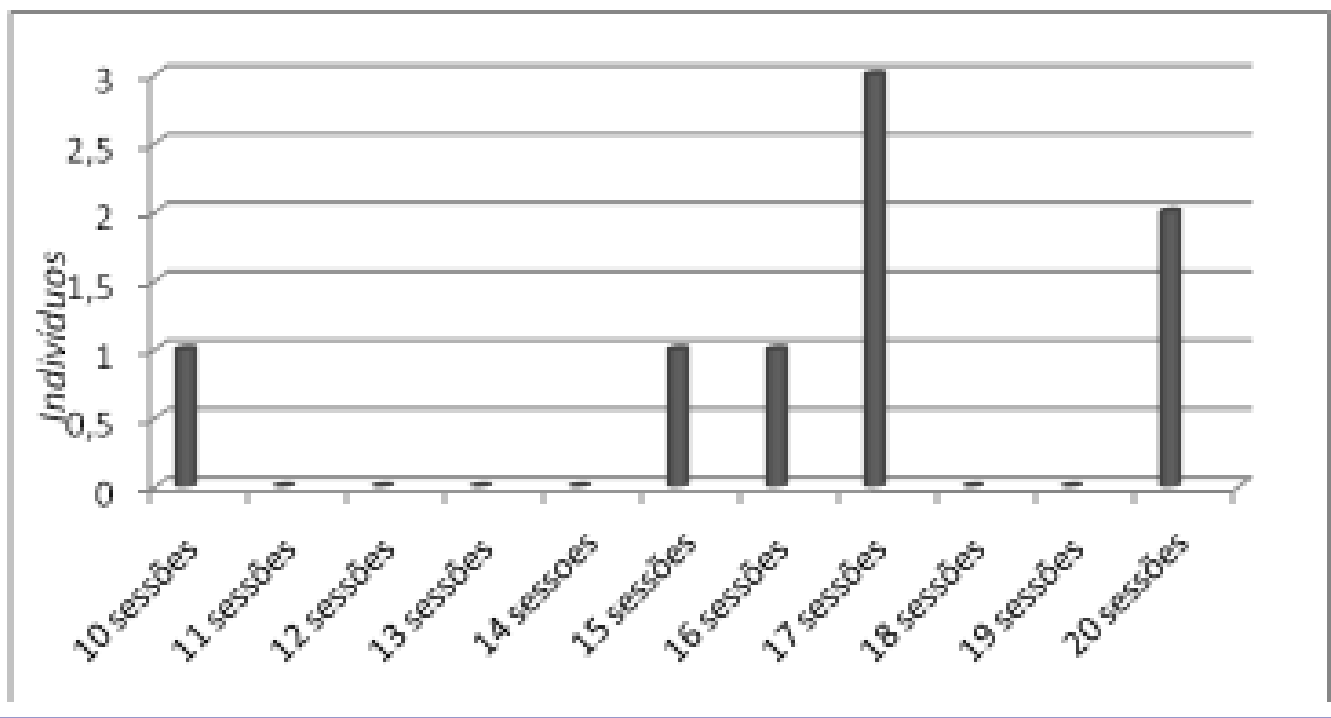

Figura 2: Distribuição do número de sessões para adequação das habilidades auditivas, por indivíduo (N=8). 
comum em meninos ${ }^{25}$. Quanto à faixa etária, observou-se que a média de idade era de oito anos, época em que a criança dedica-se ao ensino fundamental e ao processo de alfabetização.

O número de sessões semanais, realizadas com os indivíduos do estudo, variou de 10 a 20, com duração de 40 minutos cada (Figura 2). Este estudo está em conformidade com o trabalho de Kozlowski et al. ${ }^{15}$, no qual foram realizadas 16 sessões de TA semanais, portanto, 16 semanas, com duração de 45 minutos cada. Entretanto, neste estudo também evidenciouse a necessidade da continuidade do trabalho fonoaudiológico para outros sujeitos, por mais três meses, para adequar todos os aspectos do desenvolvimento. $\mathrm{Na}$ literatura, foram encontrados estudos cujos resultados indicaram que um percentual de indivíduos $\left(28,57 \%\right.$ para Beck, Calichman; Gandra ${ }^{14} ; 15 \%$ para Ribas; Rosa; Klagenberg, ${ }^{25}$; 36,66\% para Zalcman; Schochat ${ }^{8} ; 27,6 \%$ para Alonso; Schochat ${ }^{13}$ ) manteve uma ou mais dessas habilidades alteradas, mesmo após o TA. Em outro estudo ${ }^{17}$ evidenciou-se que as 10 sessões pré-estabelecidas não foram suficientes para adequar todas as necessidades de alguns indivíduos (com alterações de linguagem oral e escrita associadas), indicando a necessidade de número maior de sessões de TA.

Neste estudo, também se evidenciou essa variação de tempo para a aquisição de cada habilidade, como demonstrado na Tabela 1 e Figuras 1 e 2. No grupo estudado nesta pesquisa, observou-se que fatores ambientais, como envolvimento familiar e colaboração da equipe escolar e o processo inerente a cada indivíduo ("o tempo de cada um"), associados à sistematicidade do processo terapêutico podem ter colaborado para a variação de aquisição das habilidades auditivas propostas.

A sistematicidade do processo terapêutico é de fundamental importância, pois diversos trabalhos sobre plasticidade auditiva ${ }^{26}$ demonstram que a estimulação frequente e intensiva é fundamental. Na Tabela 1, observa-se o tempo médio de sessões para adequação de cada habilidade auditiva trabalhada nos oito indivíduos do estudo. O primeiro passo de um programa de TA requer que as habilidades alteradas sejam hierarquizadas de acordo com o padrão do desenvolvimento auditivo normal, ou seja: detecção, sensação, discriminação, localização, reconhecimento, compreensão e memória. Em cada uma dessas etapas são trabalhadas habilidades auditivas específicas ${ }^{17}$. Neste estudo, observou-se que, para as habilidades auditi- vas de reconhecimento e compreensão foram as que exigiram maior número de sessões para adequação (média de 16 e 12,25 sessões, respectivamente), resultado coerente com o esperado durante a estimulação uma vez que são habilidades auditivas mais complexas. O número menor de sessões para adequação da habilidade auditiva de compreensão pode ser justificado pelo tipo de tarefa utilizado, favorecida pelo fechamento auditivo e, consequentemente, melhorando o desempenho da compreensão auditiva.

O tempo médio de sessões, para a adequação das habilidades trabalhadas neste estudo, foi de oito sessões. Esse número de sessões está de acordo com vários autores ${ }^{8,13-19}$ que utilizaram em seus trabalhos de oito a 18 sessões de TA.

$\mathrm{Na}$ análise comparativa dos resultados deste estudo com outros trabalhos, em relação às atividades realizadas, sem e com som competitivo, pode-se observar que os resultados obtidos estão em conformidade com outros estudos ${ }^{26-7}$ onde se observou que a condição de escuta competitiva tendia a ser mais difícil que a não competitiva. Assim, mais sessões seriam necessárias para adequar o processo de escuta competitiva de um indivíduo.

Os resultados aqui descritos concordaram com os encontrados no estudo de Pinheiro e Capellini ${ }^{16}$, cujo objetivo foi caracterizar e comparar o desempenho de 40 escolares na faixa etária de oito a 14 anos de idade, com e sem distúrbio de aprendizagem em testes de PA, após a aplicação do programa de TA. Os indivíduos foram submetidos a 18 sessões de treinamento, 50 minutos cada, duas vezes por semana e foi observado que os grupos submetidos ao TA apresentaram desempenho superior àqueles dos grupos não submetidos. É importante salientar que o tempo de TA proposto é suscetível às influências de fatores individuais, sociais e ambientais, como motivação, insegurança, atenção, memória, aprendizagem, convívio familiar, características de personalidade e da própria tarefa a ser realizada e condições em geral, entre outros, que podem ocasionar maior variação no tempo médio de aquisição de cada habilidade, de acordo com o desempenho de cada indivíduo.

De acordo com os resultados demonstrados na Figura 1, nota-se que o desempenho de cada um dos oito indivíduos, referentes a cada habilidade auditiva, foi bastante heterogêneo, tanto no que se refere à quantidade de sessões necessárias para aquisição de cada habilidade como no que diz respeito aos fatores que podem interferir na realização do TA. 
Alguns indivíduos do presente estudo com DPA apresentaram, também, dificuldades escolares, como é o caso dos indivíduos 1, 2, 6 e 7, que apresentaram, respectivamente, dificuldades na escrita, dificuldade em consciência fonológica, trocas surdas sonoras e dificuldade em leitura e escrita. Embora não tenha sido o objetivo do presente trabalho, diferentes estudos ${ }^{15-7,15,28}$ revelaram que DPA frequentemente coexiste com problemas de linguagem, dificuldade de aprendizagem da leitura e escrita em sala de aula e atrasos no desenvolvimento da linguagem e que os indivíduos podem ser beneficiados quando estratégias de intervenção e estratégias compensatórias são combinadas, em prol de seu desenvolvimento. Do mesmo modo, no presente estudo, além da estimulação da função auditiva, por meio do TA, foi realizada orientação aos pais e educadores, a fim de melhorar a percepção auditiva dos indivíduos com as dificuldades acima citadas.

Entre outros fatores que podem contribuir no tempo médio de aquisição de cada habilidade, podendo ocasionar rendimento menor por parte dos indivíduos, estão as condições de saúde, como gripes, resfriados e viroses, presentes em algum momento durante a realização do TA nos indivíduos 6 e 7; condições familiares, como separação e intolerância dos pais, falta de preocupação com os filhos e dificuldade para comparecer ao atendimento, o que ocorreu com os indivíduos 3, 4 e 7; condições alimentares, como o ocorrido com o indivíduo 2 , que compareceu a algumas sessões com fome, sem ter almoçado, o que ocasionou desempenho insatisfatório durante essas sessões; falta de estimulação ambiental e reforço por parte da família, condições essas observadas como parte da dinâmica familiar dos indivíduos 4 e 7; características pessoais, como comportamento hiperativo, impulsividade, dispersão, podem estar associadas ao déficit de atenção e hiperatividade, características essas dos indivíduos 3, 4 e 5; queixas escolares dos indivíduos 3, 4 e 5, como mau comportamento, agressividade, atividades escolares que não são realizadas por eles e faltas frequentes, que podem influenciar, de maneira negativa, o desempenho das atividades realizadas durante o TA, entre outros.

Em relação aos indivíduos 1 e 5, esses foram considerados os mais interessados e colaboradores, notando-se melhora no desempenho escolar e comportamental dessas crianças. Além disso, houve grande interesse nas atividades e envolvimento da família. Kozlowski et al. ${ }^{15}$ e Samelli e Mecca ${ }^{17}$ concordam que o TA não pode ocorrer separado da família e da escola, e o apoio e participação desses é fundamental durante todo o processo terapêutico. Durante a intervenção realizada nos indivíduos deste estudo, somada ao trabalho realizado pelas fonoaudiólogas, atividades relacionadas com o que estava sendo feito em terapia foram passadas para as crianças, o que está de acordo com vários trabalhos $7,8,17$.

O programa de TA, descrito na literatura, segundo Samelli e Mecca ${ }^{17}$, foi organizado com base em estudos de diversos autores e incluiu técnicas como considerar a complexidade de tarefas dentro de cada habilidade e hierarquizar as habilidades auditivas e consideraram que, para que isso seja cumprido, todas as atividades realizadas devem ser registradas e os acertos pontuados, corroborado neste estudo, tanto em relação às técnicas utilizadas quanto em documentar os resultados do plano de intervenção, podendo incluir, segundo as recomendações da American Academy Of Audiology ${ }^{28}$, índices de desempenho auditivo, os índices funcionais de metalinguagem e/ ou medidas mais globais de escuta e comunicação, como autoavaliação.

Vale ressaltar que a amostra coletada neste estudo foi não probabilística e a análise descritiva, sendo tais resultados não passíveis de generalização. Estudos com maior casuística são fundamentais para serem alcançados marcadores de tempo para aquisição de habilidades auditivas, nesse segmento populacional, assim como desenvolver instrumentos para mensurar fatores que interferem indiretamente para o desenvolvimento das habilidades auditivas, contribuindo, assim, para o processo de intervenção, evolução e alta.

Faz-se necessário considerar e orientar recomendações a respeito da frequência necessária e suficiente, intensidade, duração das sessões e planejamento sistematizado dos programas de treinamento auditivo, considerando aspectos pessoais, sociais, ambientais, escolaridade, entre outros.

\section{Conclusão}

Todos os indivíduos estudados atingiram a adequação das habilidades auditivas propostas, num tempo médio de oito sessões. Houve variação de tempo para cada habilidade trabalhada, sendo que a habilidade auditiva que necessitou de menor número de sessões (média 2,87) foi a de localização e a maior (média 16 sessões) a de reconhecimento. 


\section{Referências}

1. Pereira ID. Sistema auditivo e desenvolvimento das habilidades auditivas. In: Ferreira LP, Befi-Lopes, DM, Limongi SCO. Tratado de Fonoaudiologia. São Paulo: Roca; 2004, p. 54752.

3. Boothroyd A. The sense of hearing. In: Boothroyd A (Ed). Speech acoustics and perception. Austin: The Pro-ed Studies in Communicative Disordes; 1986, p. 65-73.

4. Kelly BR, Davis, Hedge MN. Assessing auditory problems. In: Clinical Methods and Practicum in Audiology. California: Singular PublishingGroup; 1994, p. 179-211.

5. Musiek FE, Geurkink NA. Auditory perceptual problems in children: considerations for the otolaryngologist and audiologist. Laringoscope 1980; 90: 962-71.

2. Katz J. Tratado de Audiologia Clínica. 4ª ed. São Paulo: Manole; 1999.

6. Philips DP. Central auditory processing: a view from auditory neuroscience. Am J Otol. 1995; 16: 338-52.

7. Pereira LD. Avaliação do processamento auditivo central. In Lopes Fh O. Tratado de Fonoaudiologia. São Paulo: Roca; 1997, p. $109-26$

8. Zalcman TE, Schochat EA. Eficácia do treinamento auditivo formal em indivíduos com transtorno de processamento auditivo. Rev Soc Bras Fonoaudiol. 2007; 12: 310-14.

9. Grafman J. Conceptualizing functional neuroplasticity. J Commun Disord. 2000; 33: 346-55.

10. Tremblay KL, Kraus N. Auditory Training induces assymmetrical changes in cortical neural activity. J Speech Lang Hear Res. 2002; 45: 564-72.

11. Azevedo MF, Pereira LD. Terapia para desordem do processamento auditivo central em crianças. In: Pereira LD, Schochat E. Processamento auditivo central - manual de avaliação. São Paulo: Lovise; 1997, p.69-77.

12. Almeida SMVT, Poli IA. Balizadores do tempo de tratamento aprimoram atuação do Fonoaudiólogo. Jornal do Conselho Federal de Fonoaudiologia 2008; 39: 8-11.

13. Alonso $\mathrm{R}$, Schochat $\mathrm{E}$. The efficacy of formal auditory training in children with (central) auditory processing disorder: behavioral and electrophysiological evaluation. Braz J Otorhinolaryngol. 2009; 75: 726-32.

14. Beck CS, Calichman F, Gandra LPF. Estimulação do processamento auditivo central em crianças escolares de 7 a 10 anos de idade. Pró-fono. 1996; 8: 45-50.

15. Kozlowski $L$ et al. A efetividade do treinamento auditivo na desordem do processamento auditivo central: estudo de caso. Rev Bras Otorrinolaringol. 2004; 70: 427-32.
16. Pinheiro FH, Capellini SA. Desenvolvimento das habilidades auditivas de escolares com distúrbio de aprendizagem, antes e após treinamento auditivo, e suas implicações educacionais. Psicopedagogia. 2009; 26: 231-41.

17. Samelli AG, Mecca FDN. Treinamento Auditivo para transtorno do processamento auditivo: uma proposta de intervenção terapêutica. Rev CEFAC 2010; 12: 235-41.

18. Santos JN, Couto IC, Amorim RMC. Treinamento auditivo em oficinas: opção terapêutica grupal. Pró-fono. 2006; 18: 293302.

19. Schochat E, Musiek FE, Alonso R, Ogata J. Effect of auditory training on the middle latency response in children with (central) auditory processing disorder. Braz J Med Biol Res. 2010: 43; 777-85.

20. Pereira LD, Schochat E. Processamento Auditivo Central Manual de Avaliação. São Paulo: Lovise; 1997, p.69-77.

21. Rodrigues FMC. Processando sons I (Livro e software). $1^{\text {a }}$ ed. Ribeirão Preto: Booktoy, ISBN: 9788590739104, 2007, p. 14.

22. Santos RM, Toffoli MB, Cardoso, APB, Drumond GP, Rosa DAR. Pedro na casa mal-assombrada- desenvolvendo habilidades auditivas. Software CTS informática, 2006.

23. Dias KZ, Gil D. Treinamento auditivo formal nos distúrbios de processamento auditivo. In: Bevilacqua MC. et al. (org.). Tratado de Audiologia. São Paulo: Santos; 2011, p. 827-844.

24. Musiek FE, Schochat E. Auditory training and central auditory processing disorders: a case study. Semin Hear. 1998;19: 357-66.

25. Ribas A, Rosa MRD, Klagenberg K. Avaliação do processamento auditivo em crianças com dificuldades de aprendizagem. Psicopedagogia. 2007; 24: 37-42.

27. Almeida CC. Semi-alfabetizados versus alfabetizados: avaliação auditiva através dos testes SSW, discriminação e consciência fonológica. [Monografia Especialização em Audiologia], São Paulo: Universidade Federal de São Paulo; 1997.

27. Machado SF. O Teste SSW: avaliação e aplicação de um instrumento no estudo da avaliação da percepção da fala. 1993. [Tese de Doutorado], São Paulo: Pontifícia Universidade Católica de São Paulo; 1993.

29. American Academy Of Audiology - Clinical Practice Guidelines. Diagnosis, Treatment and Management of Children and Adults with Central Auditory Processing Disorder. [serial online] [Cited 2010 Aug 29] Available from: www.audiology.org. 